\title{
Electromagnetic Simulation of Metallic Pipeline for Non-destructive Testing
}

\author{
Zong-Jing ZHANG ${ }^{1, a}$, Shou-Qiang MEN ${ }^{1, b,{ }^{*}}$, Lei HAN ${ }^{1, c}$ \\ ${ }^{1}$ Xi'an Technological University, Xi'an 710021, China \\ a1140693381@qq.com, bmenshouqiang@xatu.edu.cn, 'skyhl123@163.com \\ ${ }^{*}$ Corresponding author
}

Keywords: Magnetic Field Intensity, Electromagnetic Simulation, Nondestructive Testing.

\begin{abstract}
The magnetic field intensity distribution near a circular defect of a metallic cylinder was computed using finite element analysis. It is found that there is an obvious interference to the local magnetic field intensity when the pipeline with a defect was placed inside a Helmholtz coil, which provides information on designing magnetic field sensor array for non-destructive testing of the defect.
\end{abstract}

\section{Introduction}

Pipeline plays a main role in transporting crude oil and natural gas. Hence, its safety attracts much attention [1-3]. At present, there are lots of non-destructive testing techniques applied to detect cracks, corrosive cavities and other defects of the pipelines, among which electromagnetic testing and ultrasonic testing are two mostly used techniques.

In this paper, a metallic pipeline model was set up. There is a round hole on the wall of the pipeline, and the pipeline was placed into a Helmholtz coil [4]. When a direct current was imposed on the coil, a uniform magnetic field in the central zone of the coil was generated. In this condition, a perturbation to the local magnetic field near the hole was introduced. By finite element analysis, the magnetic field distribution near the hole was obtained [5]. It is found that the local magnetic field changes dramatically, and the magnetic lines are not parallel to the central axis any longer, but around the hole. The simulation results cannot only predict the magnetic field perturbation near the defect, but also provide theoretical guidance to the design of magnetic field array to carry out measurement of the magnetic field distribution.

\section{Simulation Results and Discussions}

\section{Simulation Model}

In the simulation model, the material of the metallic pipeline is steel, whose relative magnetic permeability is 4000 , the electrical conductivity is $1.12 \times 10^{7}[\mathrm{~S} / \mathrm{m}]$, and the relative dielectric constant is 1 . The inner and outer diameter of the pipeline is $400[\mathrm{~mm}]$ and $600[\mathrm{~mm}]$, respectively, and its length is $500[\mathrm{~mm}]$.

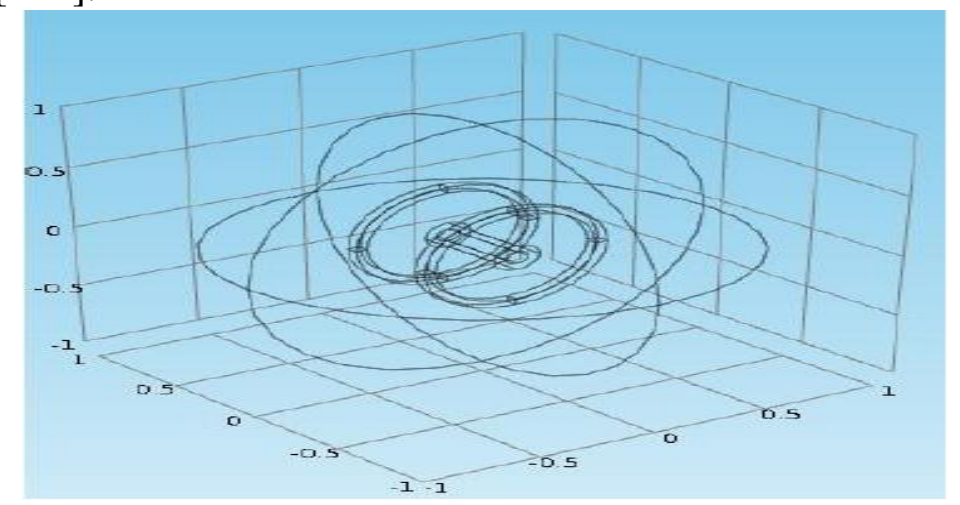

Fig. 1 Schematic of the cylinder inside the Helmholtz coil 


\section{Simulation of the Magnetic Field}

Firstly, mesh was generated, and in the steel domain, mesh generation were dense in order to demonstrate more details of the key object. Besides, the boundary condition was set to be magnetization, and the magnetic potential at the boundary is 0 .

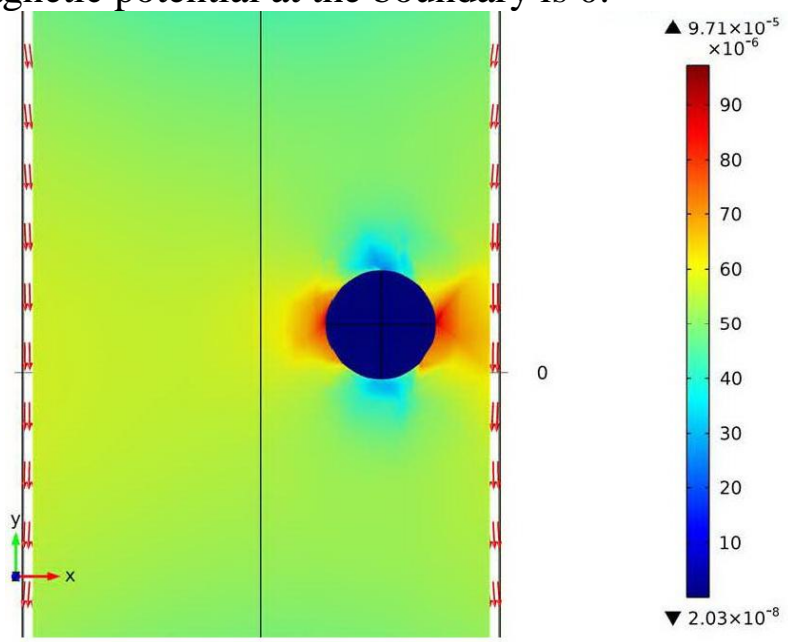

Fig. 2 Magnetic field distribtion near the round hole

When the surface electrical current was $1000\left[\mathrm{~A} / \mathrm{m}^{2}\right]$, the magnetic flux density distribution near the round hole was computed and was shown in Fig. 2. Colors in the figure imply the magnitude of the magnetic field. It can be seen that the magnetic field is nearly uniform far from the hole, which is about $50[\mu \mathrm{T}]$, whereas the local magnetic field near the surface of the round hole changes dramatically, its magnitude is changing from $40[\mu \mathrm{T}]$ to $97[\mu \mathrm{T}]$. Besides, since the matter is air inside the hole, whose relative magnetic permeability is only $0.1 \%$ of the steel, the inner magnetic flux density is only $0.02[\mu \mathrm{T}]$.
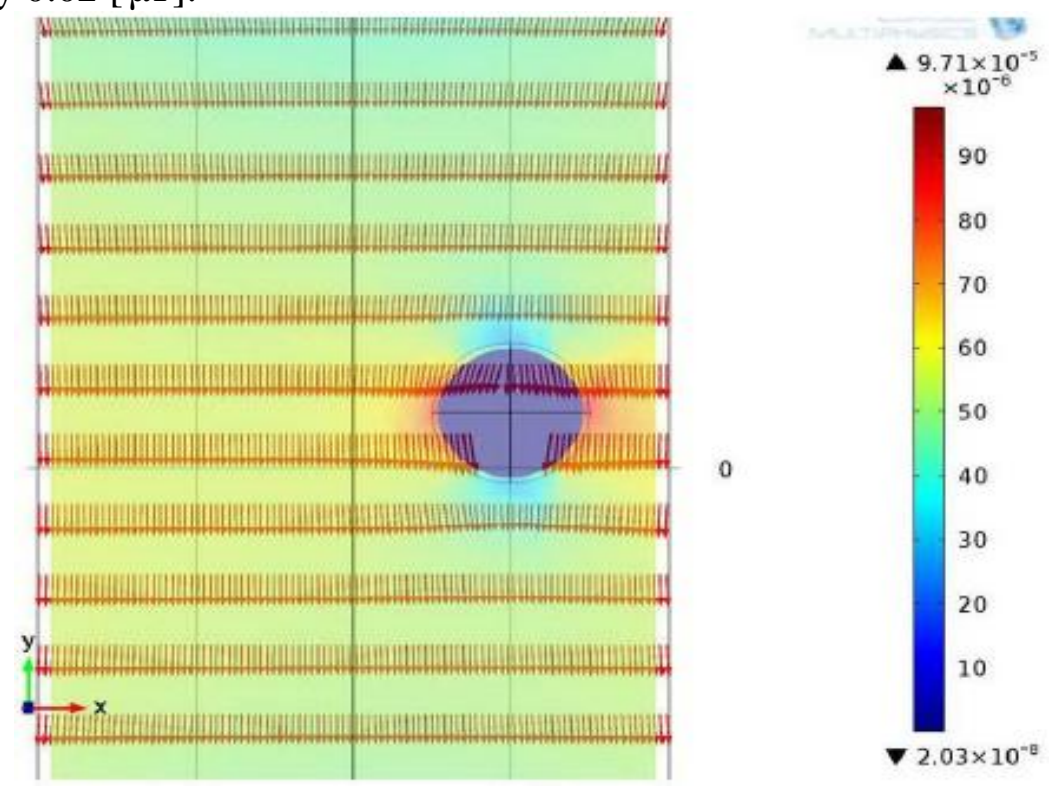

Fig. 3 Magnetic field distribution in X-z plane

In order to demonstrate the magnitude and direction simultaneously, magnetic lines were also plotted as shown in Fig. 3. This figure shows that the direction of the magnetic flux density is nearly equal to each other and the direction is parallel to the central axis far from the hole. However, the magnetic lines near the hole pass around it but through the steel whose permeability is much greater than that of the air. 


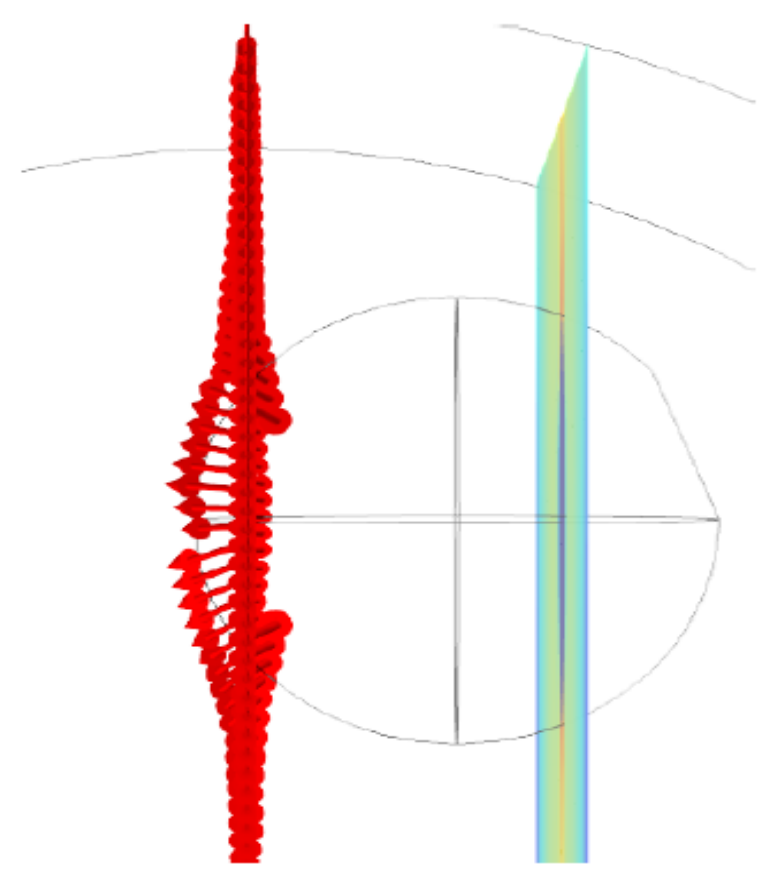

Fig. 4 Magnetic field distribution in y-z plane

The magnetic field distribution in y-z plane near the hole was depicted in Fig. 4. As can be seen, there is a strong y-component of the magnetic flux density near the hole, whereas there is only $\mathrm{z}$-component far from the defect.

According to the simulation results as shown in Fig. 3-4, one can find the magnitude and location of the magnetic field perturbation so as to judge the location of the defect. Moreover, based on the simulation, magnetic field sensors met the demand of measuring range and accuracy can be selected, and the number of the sensors and the location of each for interface reconstruction of the defect can be computed. In other words, the magnetic field sensor array can be designed, which provides guidance to develop magnetic field tomography.

\section{Conclusions}

A testing model with a uniform magnetic field was set up with respect to non-destructive testing problems of ferromagnetic pipelines. In the case of a round hole on the pipeline, a double-magnetic -field near the defect in comparison with that in other domain was found by finite element analysis, and the magnetic field direction altered a lot, too. The forward calculation provides not only the magnetic interference, but the theoretical guidance to design magnetic sensor array.

\section{Acknowledgement}

We are grateful to the financial support by National Student Innovation Training Program (grant number: 201310702034) and Education Department of Shaanxi Provincial Government Program (grant number: 11JK0518).

\section{References}

[1]Jun-Ming Lin, Development and New Progress in Electromagnetic Test Technology, Engineering \&Test, 51(2011), 1-5.

[2] Guang-Hai Li, Gong-Tian Shen, and He-Nian Li, Non-destructive Testing Techniques for Industrial Pipelines, Non-Destructive Testing,28 (2006), 89-93.

[3] Ji-Lin Ren, New Progress in Electromagnetic Testing. Nondestructive Inspection,2010(5), 1-4. 
[4]Xiao-Ke Yi, Manufacture and test of Helmholtz coils. Experiment Science and Technology, 10(2010), 170-172.

[5]Xin-Jie Wu, Sheng $\mathrm{Hu}$, and Rong-Rong $\mathrm{Fu}$, Calculation of sensitivity field of electrical capacitance tomography system based on COMSOL, Journal of Liaoning University,38(2011), 193-196. 